\title{
Simulataneous Formation of InGaN Nanostructures with Varying Shapes for White Light Source Applications
}

\author{
A. Gasim ${ }^{1}$, D. Cha ${ }^{2}$, T. K. $\mathrm{Ng}^{1}$, P. Bhattacharya ${ }^{3}$, and B. S. Ooi ${ }^{1 *}$

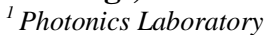 \\ King Abdullah University of Science \& Technology (KAUST), Thuwal 23955-6900, Saudi Arabia \\ ${ }^{2}$ Advanced Nanofabrication, Imaging and Characterization Core Laboratory \\ King Abdullah University of Science \& Technology (KAUST), Thuwal 23955-6900, Saudi Arabia \\ ${ }^{3}$ Department of Electrical Engineering and Computer Science, University of Michigan, 1301, Beal Avenue, Ann Arbor \\ Michigan 48109-2122, United States of America \\ *E-mail: boon.ooi@ kaust.edu.sa
}

\begin{abstract}
Varying shapes of InGaN nanostructures were simultaneously formed on silicon epitaxially. The nanowires and nanomushrooms emit violet-blue light, and broad yellow-orange-red luminescence, respectively. The combination of which is promising for white light emission.
\end{abstract}

